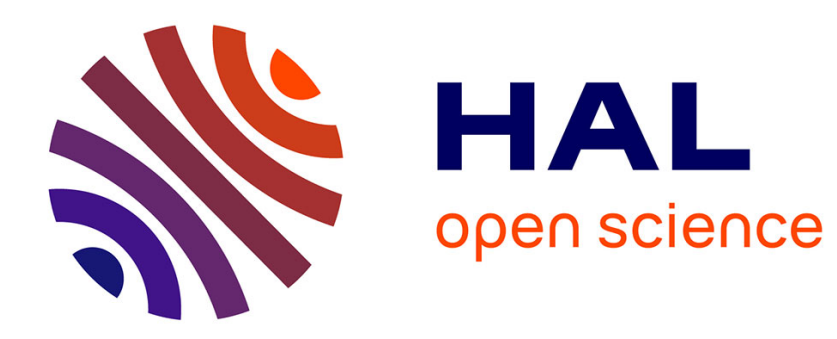

\title{
La Chine à l'heure des villes intelligentes
}

Nicolas Douay, Carine Henriot

\section{To cite this version:}

Nicolas Douay, Carine Henriot. La Chine à l'heure des villes intelligentes. L'Information géographique, 2016, 80 (3), 10.3917/lig.803.0089 . hal-01865106

\section{HAL Id: hal-01865106 https://hal.science/hal-01865106}

Submitted on 30 Aug 2018

HAL is a multi-disciplinary open access archive for the deposit and dissemination of scientific research documents, whether they are published or not. The documents may come from teaching and research institutions in France or abroad, or from public or private research centers.
L'archive ouverte pluridisciplinaire HAL, est destinée au dépôt et à la diffusion de documents scientifiques de niveau recherche, publiés ou non, émanant des établissements d'enseignement et de recherche français ou étrangers, des laboratoires publics ou privés. 


\title{
La Chine à l'heure des villes intelligentes
}

\author{
Par Nicolas Douay et Carine Henriot
}

Nicolas Douay, maître de conférences en urbanisme, Université Paris-Diderot, UMR Géographie-Cités, UMIFRE C ntre d'études français sur la Chine contemporaine - nicolas.douay@gmail.com

Carine Henriot, maître de conférences en urbanisme, Université de technologie de Compiègne, EA Avenues, et chercheur associé à l'UMR Prodig - carine.henriot @utc.fr

\section{- Introduction}

La croyance dans les opportunités offertes par la technique pour mieux aménager et gérer les villes est ancienne. Aujourd'hui, la smart city qui tient ses origines des opportunités offertes par l'exploitation des ressources numériques en est la version contemporaine. Les définitions des smart cities sont encore imprécises et largement mouvantes dans la littérature comme dans la pratique ; toutefois, le lien avec la technologie est clair même si cela peut renvoyer à une grande diversité d'usages et de niveaux d'appropriation. Michael Batty (2013) les conçoit comme des villes structurées par la gestion instantanée des Big Data issues de la technologisation des espaces urbains et des réseaux. Pour lui, les villes deviennent smart lorsqu'elles permettent le recours au traitement informatique simultané de l'information afin de gérer et d'anticiper la dynamique des espaces, des réseaux et des populations. La smart city repose ainsi sur l'usage massif de l'informatique, de capteurs, de super-calculateurs et de l'Internet, qui permettent de connaître et de gérer la ville sur le très court terme (Batty, 2013). Pour Anthony Townsend (2014), les Big Data sont même l'outil indispensable pour permettre l'émergence de véritables smart cities, structurées par une connaissance de la ville actualisée en temps réel et une forme d'ubiquité permanente.

Le caractère mouvant, voire flou, de la définition de ces smart cities permet de l'associer à différentes mutations de nos territoires. Tout d'abord, celles-ci participent à une logique capitaliste qui perpétue la croissance économique en fournissant de nouveaux marchés aux plus grands groupes privés (IBM, Cisco, Veolia, Dassault, General Electric, Siemens, Phillips...). Toutefois, par l'usage de nouvelles technologies de l'information et de la communication, 
elles visent l'amélioration de la qualité de vie et la résolution des problèmes environnementaux (Scholl et Scholl, 2014 ; Caragliu et al., 2009 ; Giffinger et al., 2007). Ainsi, la transition numérique est souvent associée à la transition environnementale. La contribution de la smart city à l'achèvement de la durabilité urbaine ne se limite pas à l'amélioration des infrastructures, puisqu'il s'agit également d'encourager les habitants à adopter des modes de vie plus soutenables en modifiant leurs comportements relatifs à la mobilité, à l'usage de l'énergie et au traitement des déchets (Khansari et al., 2013). Une smart city serait alors une ville numérique et durable où le recours au numérique permettrait d'atteindre des objectifs vertueux en intégrant, par l'optimisation de son fonctionnement, les objectifs du développement durable (Eveno, 2015).

Pour illustrer cette numérisation des villes et des politiques urbaines nous proposons d'étudier les cas de la Chine continentale et de la région administrative spéciale de Hong Kong. Notre problématique vise à replacer ces transformations contemporaines dans le temps long des dynamiques d'aménagement afin d'interroger les configurations d'acteurs et le contenu des politiques d'aménagement. Nous émettons l'hypothèse selon laquelle face à l'essoufflement des politiques de durabilité, l'intelligence urbaine correspond plutôt à l'ajout d'une dimension numérique à des projets urbains existants qui ont pu être présentés comme étant durables. Dans ce sens, au-delà des modifications réelles liées au développement de la technologie, il s'agit d'un positionnement largement stratégique qui relève beaucoup du marketing urbain de collectivités territoriales, dans un contexte de coalition de croissance, associant acteurs privés et publics, qui ne changent pas fondamentalement la nature des politiques urbaines, même si celles-ci bénéficient d'un nouveau « vernis » moderne, numérique, durable - en un mot - intelligent.

\section{- Circulation internationale de l'expertise vers la Chine}

\section{Circulation des concepts et appropriation du vocabulaire par les acteurs publics}

Les politiques urbaines circulent à l'échelle du globe mais elles ne sont pas reproduites en totalité et servent plutôt de référence dans des situations d'adaptation d'un espace à un autre (Peyroux, 2014). Les notions de « Policy Transfer $\gg$ (Dolowitz et Marsh 1996 ; Dolowitz et Marsh, 2000) ou de mobilité des politiques urbaines «Urban policies mobility» renvoient ainsi à un processus par lequel le savoir ou les connaissances sur les politiques et les arrangements administratifs et institutionnels utilisés dans un système 
politique donné (passé ou présent) sont utilisés pour développer des politiques et des arrangements dans un autre contexte. Les échanges s'appuient sur différentes communautés d'esprit, de pratiques ou d'expertises (Peck et Theodore 2010 ; Stone, 2004).

La notion de ville intelligente arrive en Chine continentale et à Hong Kong sous sa désignation anglophone « Smart City » dans la seconde moitié des années 2000, avant d'être appropriée et que cette notion ne soit sinisée, au début des années 2010. La traduction officiellement retenue est alors : «une ville intelligente » (zhihui chengshi, 智慧城市). Au-delà de cette traduction, d'autres termes apparaissent pour désigner sensiblement le même type de projet, comme «Smarter City », «Smart Green City », «Smart Pilot City » ou «Digital City ». Ces différentes terminologies fluctuent selon les configurations d'acteurs mobilisés, et l'acceptation que chacun de ces acteurs a de la ville intelligente.

\section{Le rôle des acteurs privés dans l'introduction de modèles de ville intelligente}

Dès 1998, IBM aide le gouvernement de Hong Kong à concevoir sa stratégie «Digital $21 »^{1}$ et participe ainsi à la constitution d'un marché de la ville numérique, et l'investit, en positionnant ses intérêts au cœur des politiques urbaines locales, ce qui lui permet d'être implanté lorsque la ville intelligente et ses nouveaux acteurs émergent.

En Chine continentale, à l'heure où la notion de développement durable commence seulement à percoler dans le vocabulaire officiel et les projets d'aménagement chinois (Douay et Cory, 2014), IBM lance en 2008 un plan intitulé « De la percée de la ville intelligente en Chine » (Breakthrough of smart city in China). C'est alors par l'usage d'une terminologie anglo-saxonne que cette entreprise internationale choisit d'investir le marché chinois. En 2009, IBM organise un forum à Pékin structuré autour du slogan « éclairer une planète intelligente, construire une Chine intelligente ». Les accords sont immédiatement suivis de la mise en place de «clouds $»^{2}$ pilotes dans cinq villes chinoises : Pékin, Shanghai, Shenzhen, Hangzhou et Wuxi.

\footnotetext{
1. Site officiel du plan hongkongais Digital 21, http://www.digital21.gov.hk/eng/

2. Le cloud computing ou l'informatique en nuage est l'exploitation de la puissance de calcul et de stockage de serveurs informatiques en réseau grâce à Internet.
} 


\section{Le rôle des projets de coopération internationale dans la mise en œuvre des projets}

\section{La coopération entre EU et Chine : construction de référentiels et sélection des sites pilotes}

En Chine continentale, la structuration du modèle de ville intelligente s'effectue à travers une coopération avec l'Union européenne. Fin 2011, un accord entre la commission européenne et le ministère chinois de l'Industrie et des technologies de l'information (Ministry of Industry and Information Technology - MIIT) est signé. Celui-ci vise à développer une « Green Smart City ». La coopération vise à établir un référentiel sino-européen d'expertise sur la ville intelligente. Plus concrètement, des villes pilotes sont sélectionnées en Chine et en Europe, des indicateurs qualitatifs et quantitatifs sont élaborés et validés de part et d'autre, puis chaque initiative est l'objet d'une fiche synthétique permettant de mettre en évidence ses principales caractéristiques et de les comparer. En 2013, les coopérations d'experts techniques permettent la réalisation d'une étude comparative et la publication en 2014 d'un Livre blanc (EU-China, 2014).

\section{Le projet franco-chinois de Wuhan}

Pour illustrer cette collaboration et la circulation d'une expertise qui se coconstruit à l'échelle internationale, nous pouvons pointer l'exemple du Grand Wuhan, lequel correspond à un site pilote désigné pour mettre concrètement en place cette coopération. Depuis 2010, le Grand Wuhan est devenu le site témoin de la coopération franco-chinoise dans le domaine du développement urbain durable et de l'environnement, affichant une forte mobilisation, tout au moins médiatique, des responsables politiques des deux pays. Le projet franco-chinois engagé par les deux gouvernements consiste à concevoir, dans le district de Caidian, situé dans la banlieue sud-ouest de Wuhan, une ville nouvelle d'une trentaine de kilomètres carrés planifiée pour accueillir 100000 habitants.

Les entreprises françaises mobiliseront leur expertise et leurs innovations pour réaliser ce projet de ville nouvelle qui doit servir de vitrine au savoirfaire français. C'est le cas notamment d'EDF, qui devrait contribuer à faire de l'hôpital de Zhongnan un établissement efficace au plan énergétique. L'AREP, entreprise filiale de la SNCF, et les ingénieurs de Burgeap se penchent sur les aspects environnementaux. Sur le plan des transports, c'est Keolis, groupe de transports de voyageurs détenu à $70 \%$ par la SNCF, qui travaille sur les questions d'intermodalité.

La participation des entreprises françaises est visible également au plan architectural, puisque le cabinet Arte Charpentier sera par exemple en charge de la 
création d'un nouvel hôpital. Schneider Electric doit proposer de nouvelles approches de la ville intelligente. Enfin, plusieurs dizaines d'entreprises françaises se montrent attentives aux appels d'offres qui seront lancés suite aux études préliminaires.

\section{- Processus de réception et adaptation en Chine et à Hong Kong}

\section{Une démarche descendante : la programmation centrale du gouvernement chinois}

En Chine continentale, la politique des villes intelligentes se caractérise par son caractère descendant, lequel correspond à l'organisation institutionnelle et à la pratique de l'aménagement. La politique chinoise de ville intelligente débute par une planification d'échelle nationale orchestrée par le gouvernement central. Le ministère chinois de l'Industrie et des technologies de l'information (MIIT) promeut, à la fin de l'année 2011, des programmes quinquennaux de développement de l'industrie de la sécurité de l'information, de l'Internet et du E-commerce dans le cadre de l'accord avec la Commission européenne sur les « Green Smart City ».

En 2012, le MIIT et la Commission pour les réformes et le développement (National Development and Reform Commission - NDRC) conçoivent et diffusent un document national promouvant le développement de villes intelligentes. Un avis est formulé quant à la réalisation de sites pilotes nationaux de Smart Cities, avec la mise en avant de mesures provisoires et transitoires, pour démarrer les applications concrètes à l'échelle de districts et de villes. Le MIIT choisit de développer deux sites pilotes : Yangzhou et Changzhou. Parallèlement, le ministère pour la Science et la technologie (Ministry of Science and Technology - MOST) organise, en 2012, une alliance dédiée aux villes intelligentes, la « China Smart City Industry Alliance », et promeut deux sites pilotes : Shenzhen et Wuhan.

En 2012, 2013 et 2014, la programmation change d'échelle avec la publication de listes des sites pilotes de «villes intelligentes » (zhihui chengshi, 智慧城市) planifiées par le ministère en charge des questions d'aménagement : le ministère du Logement et du développement urbain et rural (Ministry of Housing and Urban-Rural Development - MOHURD).

À la suite d'un appel d'offres, 90 collectivités territoriales chinoises sont sélectionnées en 2012, puis 103 en 2013 et enfin 84 en 2014. Ce programme vise à diffuser les politiques de villes intelligentes dans les mégapoles, les grandes villes, mais également les villes moyennes chinoises, et ce, 
Fig. 1 : Les sites pilotes chinois de villes intelligentes planifiées par le MOHURD

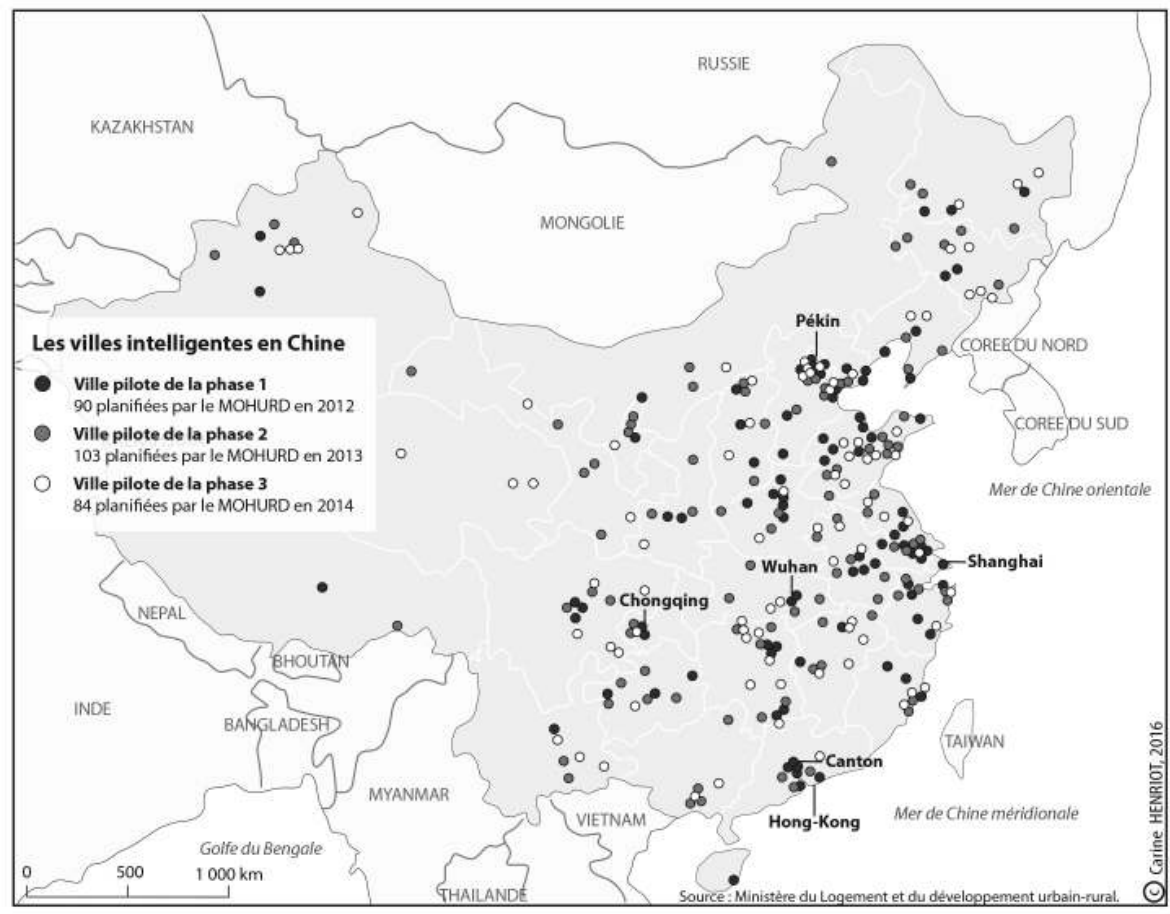

sur l'ensemble du territoire ; à renforcer la capacité de gestion des villescentre au regard de leur périphérie ; à promouvoir les TIC comme outil de gestion urbaine, l'écologie et le bas-carbone comme nouveau mode de développement. La mise en œuvre s'appuie sur l'implantation, à Pékin, d'un nouveau Centre national pour l'Innovation mais fait aussi appel à la mobilisation de différents acteurs au niveau local, notamment pour financer ces projets par le partenariat public-privé.

Au total, ce sont quatre acteurs du gouvernement central chinois qui interviennent dans la programmation des politiques de villes intelligentes et leur diffusion au plan national : le ministère de l'Industrie et des technologies de l'information (MIIT) qui a initié la coopération ente l'Union européenne et la République populaire de Chine, la Commission pour les réformes et le développement (National Development and Reform Commission - NDRC) qui intervient pour guider le MIIT dans ses premières actions, ajouter certains enjeux dans le giron des villes intelligentes, comme la question des transports et mobilités, puis articuler les différents ministères ; le ministère pour la Science et la technologie (Ministry of Science and Technology - MOST) 
qui se positionne également sur le marché de la ville intelligente ; avant que le ministère du Logement et du Développement urbain et rural (Ministry of Housing and Urban-Rural Development - MOHURD) ne programme et diffuse à grande échelle ces politiques de villes intelligentes, à travers l'implantation de sites pilotes dans toute la Chine.

En 2014, renforçant ces différentes politiques ministérielles, un document d'orientation national intitulé « National planning on new urbanisation (2014-2020) », réaffirme la poursuite des politiques et la construction de villes intelligentes, opportunité dont les collectivités territoriales se saisissent.

\section{Des coalitions de croissance locales}

Le cas de Hong Kong : le numérique au cœur des enjeux de la coalition de croissance

Selon une enquête du Boston Consulting Group de 2011 5,9 \% du PIB de la région administrative spéciale de Hong Kong relève de l'économie de $l^{\prime}$ Internet ce qui la place au $3^{\mathrm{e}}$ rang mondial derrière la Silicon Valley et New York. Le numérique est donc au cœur de l'ambition de positionner l' ancienne colonie britannique en world city asiatique dans le contexte d'une économie post-industrielle, où les industries ont été délocalisées vers le delta de la rivière des Perles, de l'autre côté de la frontière et remplacée par le secteur tertiaire.

Au quotidien, l'usage des technologies numériques est important. Nous pouvons prendre l'exemple de la carte Octopus qui sert de moyen de paiement dans les transports publics, le stationnement, les supermarchés et un nombre important de services. 28 millions de cartes sont en circulation et $99 \%$ des habitants de 15 à 64 ans en possèdent au moins une avec 13 millions de transactions chaque jour.

Le soutien aux nouvelles technologies est inscrit à l'agenda politique depuis de nombreuses années avec la stratégie « Digital $21 »$. Celle-ci a été élaborée avec l'aide d'IBM en 1998 puis régulièrement mise à jour $(2001,2004,2008)$ et fait même actuellement l'objet d'une consultation publique. Cette politique s'intitule « Smarter Hong Kong, Smarter Living » et vise l'ensemble des secteurs des politiques publiques. Plus concrètement, le gouvernement s'est engagé à :

- développer 20000 points de connexion au Wifi ;

- libérer les données publiques pour soutenir l'innovation économique ;

- numériser les activités du gouvernement pour réduire la consommation de papier ;

- soutenir le développement des Big Data et de l'Internet des objets ; 
- mettre en place une plateforme d'identité digitale pour mieux partager informations et services avec les citoyens, notamment dans le domaine de la santé.

La situation hongkongaise se caractérise donc par une configuration alliant public et privé et une offre de services de plus en plus numérisés. Le public peut s'appuyer sur l'expertise du privé et en retour le privé peut utiliser son expertise pour faire un usage commercial des données libérées par le gouvernement. Cette alliance entre public et privé sur les enjeux du numérique participe ainsi au renouvellement et à l'extension du projet partagé par les deux parties de la coalition de croissance locale.

Le cas de Shanghai : le retour du numérique (plus que du durable) au sein des coalitions de croissance

En 2010, l'Exposition universelle, organisée par la municipalité de Shanghai, est l'occasion de promouvoir le numérique dans la ville. Si le slogan de l'Expo « Better City, Better Life » a souvent été interprété, notamment par les Occidentaux, comme la promotion du développement durable, la recherche d'une ville meilleure est plutôt interprétée par les Chinois comme l'articulation de la présence végétale et du numérique dans la ville. En effet, la zone des « Meilleures pratiques urbaines » de « l'Expo Shanghai 2010 » propose alors des solutions d'aménagement respectueuses de l'environnement comme le Pavillon de la Région Rhône-Alpes (Photo 1), une construction durable mobilisant des matériaux de gros œuvre totalement recyclables et présentant plusieurs caractéristiques innovantes, comme un assainissement de l'air intérieur par phytoremédiation et un mur extérieur végétalisé, tandis que le Pavillon de Shanghai (Photo 2) se positionne comme un démonstrateur de domotique, où l'amélioration de la qualité de vie passe par les Smart grids et les solutions numériques.

Au-delà de l'épiphénomène que représente l'organisation d'un évènement international, la Municipalité de Shanghai avait déjà lancé un programme de promotion du numérique et des TIC. Dès 2009, le programme « Three year action plan for Smart Pudong (2009-2011) » est lancé, dans l'arrondissement situé à l'Est du Huangpu, symbole du renouveau de Shanghai au début des années 1990. Le programme permet de solliciter les tissus d'entreprises implantés dans la Zone de nouvelles technologies de Zhangjiang, comme le géant chinois des technologies de la communication Huawei. Par le jeu de partenariats public-privé particulièrement rentables, pour 1 million de yuans investi par le gouvernement municipal et le gouvernement d'arrondissement de Pudong, les entreprises investissent 29 millions de yuans. Pour exemple, les sommes investies dans des projets étiquetés «smart city » ont permis de mettre en service en juillet 2015 un « parking intelligent » mobilisant la 
Photo 1 : Le pavillon Rhône-Alpes

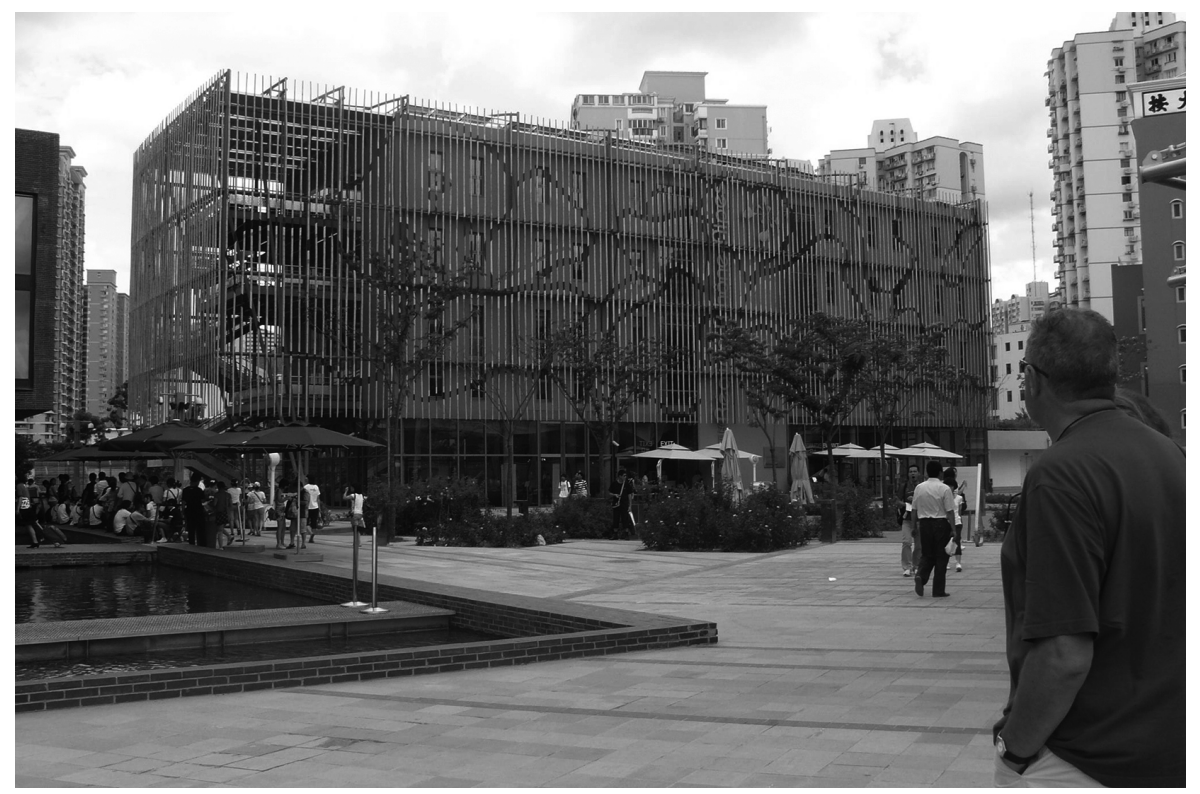

Source : C. Henriot, juillet 2010.

technologie Huawei, à Zhangjiang, au sein de l'entreprise China Unicom, pour indiquer à ses employés, via une application mobile, où ils peuvent trouver une place pour se garer. Pour mémoire, c'est déjà au sein du parc de Zhangjiang qu'avait été installé le premier réseau de vélo en libre-service de Shanghai pour effectuer le dernier kilomètre, non pas vers son domicile, mais à destination de son lieu de travail.

\section{Des projets articulant coalition de croissance locale et démonstrateur national}

La métropole chinoise littorale de Shanghai est emblématique d'un cas d'étude articulant démonstrateur de niveau national et de coalition de croissance locale. En effet, au dynamisme local encadré, dès 2009, par le «Three year action plan for Smart Pudong (2009-2011) », et réaffirmé au niveau municipal par le plan «Promoting Smart City Construction Plan (20112013)» se surimpose des initiatives relevant de l'État central. En 2012, l'arrondissement de Pudong est sélectionné pour accueillir un projet pilote correspondant à la première phase de programmation de villes intelligentes planifiée par le MOHURD. De même, en 2013, l'arrondissement de Pudong fait partie des sites pilotes retenus par le projet de coopération sino-européen « Green Smart City ». Tout dernièrement, le gouvernement d'arrondissement 
Photo 2 : Le pavillon de Shanghai (à gauche), ses murs en partie végétalisés et sa toiture photovoltaïque

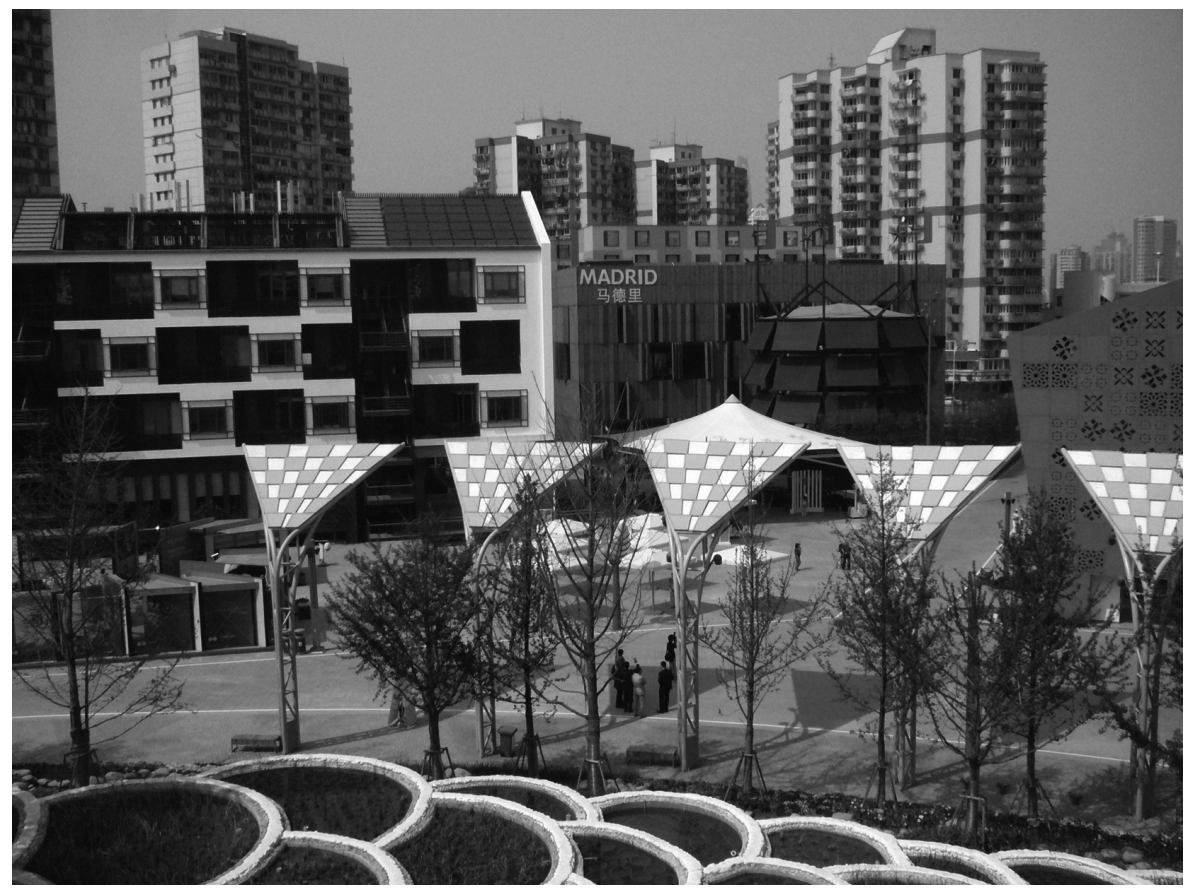

Source : C. Henriot, mai 2010.

lance le programme « iPudong 2015 », lequel s'articule autour de projets bascarbone, de transports publics intelligents, de services de santé intelligents et sur un projet de mise en place d'un e-gouvernement.

Par ailleurs, à Hangzhou, ville de taille moyenne située en Chine de l'Est, et capitale de la province du Zhejiang, au sud-ouest de Shanghai, a été sélectionnée comme étant l'une des quatre Future science and technology city du pays. Ce choix doit beaucoup à la présence d'un des principaux groupes chinois de l'économie du numérique avec le leader mondial du commerce électronique Alibaba. En coopération avec les autorités locales, ils veulent développer ce qu'ils désignent comme étant une « Silicon Valley chinoise ». Depuis 2015, un incubateur de start-up spécialisés dans les big data, le cloud, l'impression 3D et le commerce électronique a ouvert dans le cadre du projet Dream Town (Argyriou, 2016). Réalisé par des architectes internationaux et suivants les dernières innovations environnementales, ce nouveau quartier fait le lien entre le campus de l'université et une zone d'activité économique plus classique. 


\section{- De quelle ville, la smart city est-elle le nom ?}

\section{Une approche technologique de la planification?}

La première interprétation que l'on peut effectuer de l'émergence du numérique dans la pratique de la planification correspond au renforcement de la dimension technique de la fabrication de la ville. Nous pouvons illustrer cette perspective rationnelle par le projet ForCity qui propose une nouvelle manière de pratiquer l'aménagement au sein du programme collaboratif d'innovation «Wise City » porté auprès du Gouvernement de la région de Hong Kong par la Chambre de commerce française à Hong Kong avec un écosystème de grandes entreprises françaises autour du grand groupe Veolia. ForCity réalise une «maquette numérique » de Hong Kong incluant des modèles simples d'interactions entre le territoire et les métiers de Veolia : valorisation des déchets, cycle de l'eau, distribution de froid dans les bâtiments. L'enjeu est de proposer au Gouvernement de Hong Kong une nouvelle façon d'envisager sa pratique de la planification urbaine. Cela concerne notamment le développement des North East New Territories à proximité de la frontière avec la ville de Shenzhen en Chine continentale. Sur ces espaces, aujourd'hui agricoles ou naturels, des quartiers entiers vont être édifiés, des infrastructures de transport et d'autres équipements seront construits.

L'objectif est d'analyser comment ce nouveau développement va modifier l'équilibre du reste de Hong Kong afin d'anticiper ces transitions dans un espace géographique contraint, déjà soumis à une pression environnementale élevée du fait de son dynamisme économique. Pour Veolia, proposer au Gouvernement de collaborer sur la prospective de ces territoires au travers de la plateforme ForCity, «c'est inventer une nouvelle façon de valoriser ses savoir-faire auprès de son client et de concevoir des services au plus près des besoins du territoire $»^{3}$.

\section{Une approche durable de la planification?}

La deuxième interprétation possible renvoie au tournant durable de la planification urbaine en Chine. Ainsi, l'arrondissement de Pudong correspond à un projet de développement urbain prioritaire pour la municipalité de Shanghai depuis le début des années 1990. Avec la relance des réformes denguistes et l'accession à de hautes responsabilités dans l'appareil d'État de deux de ses anciens maires, Jiang Zemin et Zhu Rongji, la ville de Shanghai est désignée pour devenir la « tête du dragon » symbolisant la vallée du Yangzi et l'économie chinoise. La ville connaît une forte croissance, qui conduit à 
un redéploiement territorial dans la contiguïté de l'ancienne Puxi, à l'ouest du Huangpu, tandis que la Nouvelle Zone de Pudong, à l'est, se structure dans les années 1990 (Sanjuan, 2009). En juillet 1992 le « schéma directeur du nouvel arrondissement de banlieue de Pudong » cristallise la «modernisation de Shanghai » et planifie notamment l'aménagement de cinq zones de développement destinées à soutenir l'ensemble des champs économiques : le centre d'affaires de Lujiazui, la zone de libre-échange de Waigaoqiao, celle d'import-export de Jinqiao, le parc industriel de nouvelles technologies de Zhangjiang et le parc d'agriculture moderne de Sunqiao (Henriot, 2016).

Depuis le milieu des années 2000, le développement durable est devenu l'un des objectifs affichés dans les projets d'aménagement chinois. En 2009, dans l'arrondissement de Pudong est même programmé un nouveau secteur d'affaires, le Caohejing Kangqiao Buisness Park ${ }^{4}$, où l'intégralité des aménagements respecte et sont certifiés LEED (Leadership in Energy and Environnemental Design) un système nord-américain de standardisation de bâtiments à haute qualité environnementale. Suivant les tendances et normes de constructions internationales, les grandes entreprises sont incitées à investir le champ de l'éco-construction, voire à atteindre des objectifs bas-carbone, comme le nouveau centre de recherche L'Oréal, dessiné par Arte-Charpentier et techniquement conçu par EDRI ${ }^{5}$, pour respecter la certification LEED Gold. Ce bâtiment a été implanté à Pudong et inauguré en juillet 2015, sous le slogan Beauty for all. En favorisant un urbanisme respectueux du développement durable, le gouvernement d'arrondissement promeut dans les années 2000, le développement durable, sans toutefois en assumer le coût financier.

Au début des années 2010, les tendances et le vocabulaire évoluent ; les projets de développement urbain réintègrent le numérique et l'amalgament à la durabilité sous l'appellation «ville intelligente »: « Smart Pudong » est lancé en 2009, puis « iPudong » en 2015.

\section{Une approche marketing de la planification ?}

La troisième possibilité questionne la substance même des transformations en lien avec l'émergence de l'intelligence comme nouveau référentiel et fait l'hypothèse d'une utilisation à visée marketing dans l'optique d'un storytelling ${ }^{6}$ des politiques urbaines. En effet, même si le contenu des plans

\footnotetext{
4. Site officiel de la zone d'affaires de Kangqiao, http://bear-id.com/projects/kangqiao-business-park/

5. Présentation du projet sur le site officiel Arte Charpentier, http://www.artecharpentier.com/en/projects/1437-loreal_research_and_innovation_centre.html

6. Le storytelling correspond à un mode de communication narrative qui désigne l'art de raconter des histoires.
} 
et projets évolue lentement, les concepts utilisés pour les présenter peuvent suivre des évolutions plus rapides que l'on peut même assimiler à des modes.

Pour reprendre l'exemple hongkongais, nous pouvons noter que le terme de « ville durable » a émergé à la fin des années 1980 puis a été suivi par celui de « ville bas-carbone » et qu'il est maintenant remplacé par celui de « ville intelligente ». Cette évolution peut s'observer dans les discours politiques et les documents techniques présentant les stratégies d'aménagement. Ainsi, en janvier 2015, le chef de l'exécutif de la région de Hong Kong a insisté sur l'importance du numérique dans son discours annuel de politique générale. Plus spécifiquement, il a proposé de faire du projet urbain de l'Est de la péninsule de Kowloon un laboratoire pilote de Smart City.

Cet ancien secteur industriel de l'agglomération connaît une profonde mutation. Celle-ci est d'envergure car ce territoire accueillait l'aéroport de Kai Tak créé pendant la période coloniale britannique. Le déménagement de cette infrastructure en périphérie de la ville en 1998 libère un espace considérable, d'autant que la proximité de l'aéroport avait agrégé de nombreuses activités manufacturières et industrielles. Alors que la montée en gamme technologique des industries hongkongaises s'est accompagnée de nombreuses délocalisations en Chine continentale, de l'autre côté de la frontière dans le delta de la rivière des Perles, les terrains en mutation sont nombreux et offrent d'importantes perspectives de valorisation.

Aujourd'hui, le gouvernement souhaite faire de ce secteur assez central le deuxième Central Business District de Hong Kong ce qui permet de décentraliser un peu plus les activités économiques tertiaires qui restent largement concentrées sur l'île de Hong Kong. Cela correspond à la création de 4 millions de $\mathrm{m}^{2}$ de bureaux qui viennent s'ajouter aux espaces déjà existants pour une offre totale de 5,4 millions de $\mathrm{m}^{2}$. Du point de vue des modes de fabrication de la ville, le gouvernement se retrouve dans une situation assez inédite. En effet, traditionnellement, l'urbanisation s'appuie soit sur la création de villes nouvelles par la transformation d'anciens espaces verts ou par la création de foncier gagné sur la mer par remblais. Ici le projet est plus complexe du point de vue social, car il doit s'appuyer sur un processus de renouvellement urbain. À la différence d'autres expériences, comme le projet de Lee Tung Street à Wan Chai (Douay, 2010), la rénovation urbaine ne peut être brutale. Il convient de prendre en compte les activités économiques, principalement de la logistique, qui restent dans le quartier tout en soutenant la transition vers une diversification des activités.

La pratique de l'aménagement devient donc plus partenariale afin d'éviter les protestations des quelques résidents du quartier, des propriétaires fonciers et surtout des groupes d'intérêt à l'échelle de l'ensemble de la région. 
Dans cette perspective, le recours au concept de ville intelligente participe d'un mouvement de dépolitisation des enjeux d'aménagement pour éviter les contestations. En effet, qui peut s'opposer à une ville durable et intelligente ? Ainsi, les objectifs qui sont présentés comme relevant de cette stratégie de ville intelligente portent sur la mobilité avec le soutien aux mobilités alternatives à l'automobile, les équipements avec une attention particulière aux espaces publics et le partage des informations publiques avec le public. Plus concrètement, cette mise en scène «intelligente » permet de masquer la faible avancée du projet urbain et d'en renforcer l'acceptabilité sociale en masquant les controverses quant aux bénéficiaires du projet qui vont pouvoir profiter de la valorisation du quartier. Ainsi, même si l'usage des technologies est assez limité ou en tout cas pas plus que dans d'autres projets, la thématique numérique et intelligente est centrale dans la communication du projet apportant ainsi un storytelling efficace pour rendre le projet acceptable, donc moins controversé.

\section{- Conclusion}

La «mise en intelligence » des villes chinoises, sur le continent comme à Hong Kong, s'inscrit dans une dynamique globalisée d'inflexion des référentiels urbains, animée par des multinationales chinoises et étrangères. La réception chinoise de ce nouveau référentiel - l'intelligence urbaine - s'inscrit souvent dans le cadre de coopérations internationales, caractérisées par la mise en place de programmes descendants, où chaque niveau institutionnel ou secteur d'action de la sphère publique peut se retrouver en décalage, voire en concurrence. Localement, se dessinent alors des configurations territoriales où acteurs privés et publics coopèrent, au sein de coalitions de croissance, pour donner corps à cet engouement, certain, en Asie, pour le numérique. Les métropoles mondiales, comme Shanghai et Hong Kong, jouent, de ce fait, un rôle particulier de récepteur, de site pilote, puis de diffuseur de l'innovation vers des espaces urbains périphériques. Toutefois, lorsque l'on observe ces politiques et leur réelle mise en œuvre, il est manifeste que l'application des technologies ne change pas encore réellement la réalité des villes chinoises, dans leur matérialité, comme dans leur usage. Les politiques chinoises de villes intelligentes relèvent plutôt de stratégies de marketing urbain où l'intelligence est venue remplacer le durable, où finalement l'intelligence réalise une synthèse contemporaine des enjeux numérique et durable. Dans ce sens, la mise en intelligence des villes apparaît plutôt comme une strate supplémentaire dans la dynamique de construction des villes par le projet urbain, lequel se signale par son rôle d'assembleur (Hernandez, 2015). Au-delà du positionnement stratégique et de l'inflexion des 
référentiels urbains, un certain recul sur la mise en place de ces projets sera nécessaire, pour évaluer dans quelle mesure cette nouvelle « intelligence » des villes chinoises participe d'un développement durable sur le continent comme à Hong Kong.

\section{$\nabla$ Bibliographie}

Argyriou I. (2016), « Planning the Smart City in China : Key Policy Issues and the Case of Dream Town in the City of Hangzhou », Conférence IW3C2, 11-15 avril, Montréal.

Batty M. (2013), « Big Data, Smart Cities and City Planning », Dialogues in Human Geography, $n^{\circ} 3-3$, p. 274-279.

Caragliu A., Del BoY C. et Nijkamp P. (2009), Smart cities in Europe, Series Research Memoranda 0048. VU, University Amsterdam, Faculty of Economics, Business Administration and Econometrics.

Dolowitz D. et Marsh D. (1996), «Who Learns What from Whom : A Review of the Policy Transfer Literature », Political Studies, $n^{\circ} 44$ (2), p. 343-57.

Dolowitz D. et Marsh D. (2000), «Learning from Abroad: the role of policy transfer in contemporary policy-making », Governance, 13 (1), p. 5-24.

Douay N. et Cory T. (2014), « Développement urbain durable en Chine : entre apprentissage et mirage marketing ». in Hubert M., Lewis P. et Raynaud M. M. (dir.), Les Grands Projets urbains. Territoires, acteurs et stratégies, Montréal: Presses de l'université de Montréal, p. 239-262.

EU-China Smart and Green City Cooperation, 2014. « Comparative Study of Smart Cities in Europe and China », Current Chinese Economic Report Series, Springer, 261 p.

Eveno E. (2015), " La ville intelligente, un modèle de gestion urbaine externalisée? », Séminaire sur la Circulation des références urbaines, LabEx DynamiTe, animé par Peyroux E. et Sanjuan T, Paris, UMR PRODIG, le 30 mars 2015.

Giffinger R., Fertner C., Kramar H., Kalasek R., Pichler-Milanovic N. et Meijers E. (2007), "Smart cities : ranking of European medium-sized cities », Final report for smart-cities.eu.

Henriot C. (2016), « Métropolisation chinoise et villes nouvelles : l'exemple de l'aménagement polycentrique de Shanghai », Géoconfluences, 2016, mis en ligne le 14 février 2016.

http://geoconfluences.ens-lyon.fr/informations-scientifiques/dossiers-regionaux/lachine/corpus-documentaire/villes-nouvelles

Hernandez F. (2015), « Assembler les projets pour faire territoire. D'une exploration compréhensive des pratiques à la formalisation d'un cadre d'analyse », mémoire d'Habilitation à Diriger des Recherches, soutenu le 3 décembre 2015 à l'Institut d'Urbanisme de Grenoble.

Khansari M., Mostashari A. et Mansouri M. (2013), « Impacting Sustainable Behaviour and Planning « in Smart City » », in International Journal of Sustainable Land use and Urban Planning, Vol. 1, $\mathrm{n}^{\circ}$ 2, p. 46-61.

Peyroux E. (2014), «Circulation des modèles urbains, développement économique et géopolitique : la stratégie des relations internationales de Johannesburg » Présentation 
au séminaire « Circulation des modèles urbains », LabEx DynamiTe, animé par Peyroux E. et Sanjuan T., Paris, UMR PRODIG, le 10 février 2014.

Peck J. et Theodore N. (2010), « Mobilizing Policy: Models, Methods, and Mutations», Geoforum, ${ }^{\circ}$ 41, p. 169-174.

Sanjuan T. (2009), Atlas de Shanghai, Paris, Autrement, 88 p.

Scholl Hans J. et Scholl Margit C. (2014), « Smart Governance : A Roadmap for Research and Practice », iConference 2014 Proceedings, p. 163-176.

Stone D. (2004), "Transfer agents and global networks in the 'transnationalization' of policy », Journal of European Public Policy, Vol. 11, n 3, June 2004, p. $545-566$.

Townsend A. (2014), Smart Cities : Big Data, Civic Hackers and the Quest for a New Utopia, New York, W.W. Norton \& Company, 416 p. 